\title{
Zukunft der Pflege in Österreich
}

\author{
Der ÖGKV fordert Politik zum Handeln auf
}

Derzeit arbeiten etwa 60.000 Menschen in Österreich in Pflegeberufen. Der Bedarf, vor allem in der Langzeit- und der extramuralen Pflege, ist damit nicht gedeckt. Und die Politik hat bis jetzt keine Lösungsangebote, stellte Ursula Frohner, Präsidentin des Österreichischen Gesundheits- und Krankenpflegeverbands kürzlich fest: „Die Zukunft der Pflege in Österreich ist alles andere als gesichert.“ An die neue Bundesregierung richtete der ÖGKV Ende Oktober ganz konkrete Forderungen, um die professionelle Pflege in Österreich abzusichern.

Drei Themenbereiche bedürfen einer möglichst raschen und umfassenden Lösung:

- Der Stellenwert der professionellen Pflege in der Gestaltung der Gesundheits- und Sozialpolitik und die entsprechenden Voraussetzungen

- Besserer Zugang für alle zum Angebot der Fachpflege

- Ausbau der Ausbildung und Pflegewissenschaft.

Sowohl die steigende Lebenserwartung als auch die Zunahme chronischer Krankheiten und die steigenden Kosten des Gesundheitssystems erfordern eine bessere Einbindung der Fachpflege in die Gesundheitsversorgung der Bevölkerung. Der moderne Sozialstaat, so Frohner, geht in Sachen Pflege oft reichlich zynisch vor: Eine sehr kostspielige Hightech-Medizin hat Großartiges geleistet und kann das Überleben sicherstellen, wo dies noch vor nicht allzu langer Zeit unvorstellbar war. Kaum jedoch verlässt derselbe Patient das Spital, ist für seine weitere pflegerische Betreuung nicht gesorgt. Im extramuralen Bereich sind für eine ausreichende Pflege keinerlei Vorkehrungen getroffen.

Eine Befragung der politischen Parteien im vergangenen Nationalratswahlkampf ergab eine grundsätzliche Befürwortung einer Bildungs- und Ausbildungsreform in der Fachpflege, berichtete Frohner, wobei FPÖ und KPÖ auch die Notwendigkeit sehen die Mittel für die Pflegeforschung zu erhöhen. Die
Grünen schlagen einen Stufenplan für die Ausbildung von Pflegewissenschaftlern vor und sie sehen ebenso wie die SPÖ neue Berufsbilder und Berufsfelder als Möglichkeiten, die Fachkompetenz der Pflege zu nutzen. Eine Entwicklung, die auch finanziert werden sollte, wie die beiden Parteien feststellen.

\section{Der Forderungskatalog}

Ganz konkret nennt der ÖGKV vier Forderungen, um eine bessere Positionierung der Fachpflege und damit eine verbesserte Gesundheitsversorgung durch Einbindung der Pflege sicherzustellen.

- Erfassung des Fachpflegepotentials als Basis für seriöse Bedarfsplanung. Damit soll eine klare Trennung zwischen Fachpflege und Betreuungsdiensten erreicht werden. Die meisten EU-Mitgliedsstaaten fordern eine Registrierung von Pflegekräften. Daher fordert der ÖGKV die gesetzliche Standeslistenführung von Angehörigen der Gesundheits- und Krankenpflegeberufen.

- Etablierung der routinemäßige Pflegeberatung bei der Pflegegeldeinstufung, und die Implementierung von Gesundheitsberatung. Derzeit wird bei der Einstufung der Pflegebedürftigkeit für das Pflegegeld nicht zwischen Pflegediag-

\section{Pflegekammer}

In Deutschland wurde 2009 die Nationale Konferenz zur Errichtung von Pflegekammern in Deutschland gegründet. Eine Pflegekammer erhielte vom Gesetzgeber Hoheitsrechte und besondere Vollmachten übertragen und kann so die berufliche Selbstverwaltung und die berufsfachliche Kontrolle der Berufsangehörigen durchführen. Dazu zählen die Erlassung beruflicher Richtlinien und Vorschriften, sowie deren Umsetzung in die Praxis und die Überwachung ebenso wie ein Berufsbild, Inhalte und das Aktionsspektrum der Pflege.

Im Aufgabenbereich der Kammer läge auch die Abhaltung eines pflegerischen Staatsexamens und damit die Lizenzierung der Berufsangehörigen und eine damit einhergehende Registrierung.

Eine Pflegekammer wäre für eine einheitliche Ausbildungs- und Prüfungsordnung zuständig und für einheitliche Standards für praxisbezogene Fortbildungsmaßnahmen. Eine Zusammenarbeit der Kammer mit anderen beruflichen Organisationen könnte das berufspolitische Gewicht der Pflege verstärkt und dadurch der Durchsetzung diesbezüglicher pflegerischer Interessen Nachdruck verleihen. Experten, die im Dienste einer Berufskammer stehen, werden auch für die hoheitliche Verwaltung und die Politik zu Ansprechpartnern. So erhielte die Fachpflege einen höheren Stellenwert in der politischen Entscheidungsfindung. 